\title{
OPEN Influence of oxidative stress on vascular calcification in the setting of coexisting chronic kidney disease and diabetes mellitus
}

\author{
Shuhei Watanabe, Hideki Fujii ${ }^{\bowtie}$, Keiji Kono, Kentaro Watanabe, Shunsuke Goto \& \\ Shinichi Nishi
}

Vascular calcification (VC) is a common complication in patients with chronic kidney disease (CKD). Particularly, CKD patients with diabetes mellitus (DM) develop severe VC. Specific mechanisms of VC remain unclear; this study aimed to investigate them in the context of coexisting CKD and DM, mainly regarding oxidative stress. Sprague Dawley rats were randomly divided into six groups as follows: control rats (Control), 5/6 nephrectomized rats (CKD), streptozotocin-injected rats (DM), $5 / 6$ nephrectomized and streptozotocin-injected rats (CKD + DM), CKD + DM rats treated with insulin (CKD + DM + INS), and CKD + DM rats treated with antioxidant apocynin (CKD + DM + APO). At 18 weeks old, the rats were sacrificed for analysis. Compared to the control, DM and CKD groups, calcification of aortas significantly increased in the CKD + DM group. Oxidative stress and osteoblast differentiationrelated markers considerably increased in the CKD + DM group compared with the other groups. Moreover, apocynin considerably reduced oxidative stress, osteoblast differentiation-related markers, and aortic calcification despite high blood glucose levels. Our data indicate that coexisting CKD and DM hasten VC primarily through an increase in oxidative stress; anti-oxidative therapy may prevent the VC progression.

In patients with chronic kidney disease (CKD), cardiovascular disease (CVD) is a major cause of death ${ }^{1-3}$. The previous studies have reported that mortality resulting from CVD is higher in end-stage kidney disease (ESKD) patients than in the general population ${ }^{4}$. Vascular calcification (VC) is a type of cardiovascular lesions that is commonly observed in patients with $\mathrm{CKD}^{5}$. The prevalence and degree of VC increases with the CKD progression, and they are an independent predictor of CVD events and mortality ${ }^{6}$.

Needless to say, although CKD itself is a crucial risk factor for VC, several factors contribute to the pathogenesis of VC. Diabetes mellitus (DM) is particularly important among them. As DM is a major cause of ESKD, coexisting CKD and DM are commonly observed in clinical settings ${ }^{7}$. Moreover, patients with both CKD and $\mathrm{DM}$ are known to have higher incidences of CVD and all-cause mortality than those with either CKD or DM ${ }^{8,9}$. Considering these findings, it is important to evaluate the pathological mechanisms of VC in the setting of coexisting CKD and DM to improve their prognosis. However, to our knowledge, there is only a little information on this topic ${ }^{10}$, and the detailed mechanisms of VC in this specific context remain unclear. Particularly, there are few in vivo animal studies that involve both CKD and DM.

Oxidative stress, which is a risk factor for CKD and CVD, is known to rise in correlation with declining renal function $^{11-13}$. The prognosis and occurrence of CVD events are associated with serum oxidative stress marker levels, which are elevated in patients with advanced-stage $\mathrm{CKD}^{14,15}$. Oxidative stress has been implicated in contributing to cardiac and kidney damage. Previously, we also reported that oxidative stress plays a crucial role in aggravating the organ damage, such as in the heart, kidneys, bones, and aortas of diabetic and CKD model rats $^{16-19}$. Several experimental studies have indicated that various antioxidant approaches can attenuate the progression of organ damage in diabetic conditions ${ }^{19-21}$. 


\begin{tabular}{|c|c|c|c|c|c|c|}
\hline & \begin{tabular}{|l|} 
Control \\
$(\mathrm{N}=6)$
\end{tabular} & $\begin{array}{l}\mathrm{DM} \\
(\mathrm{N}=6)\end{array}$ & $\begin{array}{l}\text { CKD } \\
(\mathrm{N}=6)\end{array}$ & \begin{tabular}{|l} 
CKD+DM \\
$(\mathrm{N}=6)$
\end{tabular} & $\begin{array}{l}\text { CKD + DM + INS } \\
(\mathrm{N}=6)\end{array}$ & $\begin{array}{l}\mathrm{CKD}+\mathrm{DM}+\mathrm{APO} \\
(\mathrm{N}=6)\end{array}$ \\
\hline Body weight (g) & $561 \pm 9$ & $432 \pm 3^{\mathrm{a}}$ & $403 \pm 20^{\mathrm{a}}$ & $286 \pm 10^{\mathrm{a}, \mathrm{b}, \mathrm{c}}$ & $408 \pm 8^{\mathrm{a}, \mathrm{d}}$ & $298 \pm 11^{\mathrm{a}, \mathrm{b}, \mathrm{c}, \mathrm{e}}$ \\
\hline SBP (mmHg) & $120 \pm 3$ & $131 \pm 0^{\mathrm{a}}$ & $138 \pm 1^{\mathrm{a}}$ & $146 \pm 4^{\mathrm{a}, \mathrm{b}}$ & $138 \pm 2^{\mathrm{a}}$ & $138 \pm 2^{\mathrm{a}}$ \\
\hline Alb $(\mathrm{g} / \mathrm{dL})$ & $3.8 \pm 0.1$ & $3.1 \pm 0.1^{\mathrm{a}}$ & $3.4 \pm 0.1^{\mathrm{a}}$ & $2.7 \pm 0.1^{\mathrm{a}, \mathrm{b}, \mathrm{c}}$ & $3.1 \pm 0.1^{\mathrm{a}, \mathrm{b}}$ & $2.7 \pm 0.1^{\mathrm{a} a \mathrm{~b}, \mathrm{c}, \mathrm{e}}$ \\
\hline BUN (mg/dL) & $18.1 \pm 0.5$ & $21.6 \pm 0.6$ & $39.2 \pm 2.9^{\mathrm{a}, \mathrm{b}}$ & $35.8 \pm 1.0^{\mathrm{a}, \mathrm{b}}$ & $40.5 \pm 2.0^{\mathrm{a}, \mathrm{b}}$ & $30.7 \pm 2.5^{\mathrm{a}, \mathrm{b}, \mathrm{c}, \mathrm{e}}$ \\
\hline Ccr (mL/min) & $4.2 \pm 0.3$ & $5.9 \pm 0.4^{\mathrm{a}}$ & $1.1 \pm 0.1^{\mathrm{a}, \mathrm{b}}$ & $2.0 \pm 0.2^{\mathrm{a}, \mathrm{b}}$ & $1.5 \pm 0.1^{\mathrm{a}, \mathrm{b}}$ & $2.4 \pm 0.3^{\mathrm{a}, \mathrm{b}, \mathrm{c}}$ \\
\hline Blood glucose $(\mathrm{mg} / \mathrm{dL})$ & $86 \pm 3$ & $360 \pm 9^{\mathrm{a}}$ & $83 \pm 3^{\mathrm{b}}$ & $407 \pm 30^{\mathrm{a}, \mathrm{c}}$ & $108 \pm 16^{\mathrm{b}, \mathrm{d}}$ & $341 \pm 52^{\mathrm{a}, \mathrm{c}, \mathrm{e}}$ \\
\hline HbAlc (\%) & $3.2 \pm 0.1$ & $9.2 \pm 0.4^{\mathrm{a}}$ & $3.3 \pm 0.1^{\mathrm{b}}$ & $9.5 \pm 0.3^{\mathrm{a}, \mathrm{c}}$ & $3.8 \pm 0.1^{\mathrm{b}, \mathrm{d}}$ & $9.3 \pm 0.6^{\mathrm{a}, \mathrm{c}, \mathrm{e}}$ \\
\hline $\mathrm{Ca}(\mathrm{mg} / \mathrm{dL})$ & $10.2 \pm 0.1$ & $9.4 \pm 0.1$ & $7.5 \pm 0.3^{\mathrm{a}, \mathrm{b}}$ & $7.5 \pm 0.4^{\mathrm{a}, \mathrm{b}}$ & $7.7 \pm 0.3^{\mathrm{a}, \mathrm{b}}$ & $7.8 \pm 0.2^{\mathrm{a}, \mathrm{b}}$ \\
\hline $\mathrm{P}(\mathrm{mg} / \mathrm{dL})$ & $8.3 \pm 0.3$ & $9.3 \pm 0.1$ & $16.3 \pm 1.2^{\mathrm{a}, \mathrm{b}}$ & $15.6 \pm 0.7^{\mathrm{a}, \mathrm{b}}$ & $15.5 \pm 0.1^{\mathrm{a}, \mathrm{b}}$ & $13.6 \pm 1.2^{\mathrm{a}, \mathrm{b}}$ \\
\hline iPTH $(\mathrm{pg} / \mathrm{mL})$ & $974 \pm 136$ & $1357 \pm 130$ & $2937 \pm 184^{\mathrm{a}, \mathrm{b}}$ & $1762 \pm 121^{\mathrm{a}, \mathrm{c}}$ & $2914 \pm 308^{\mathrm{a}, \mathrm{b}, \mathrm{d}}$ & $1962 \pm 125^{\mathrm{a}, \mathrm{ce} e}$ \\
\hline
\end{tabular}

Table 1. Animal characteristics at 18 weeks old. DM diabetes mellitus, CKD chronic kidney disease, INS insulin, $A P O$ apocynin, $S B P$ systolic blood pressure, $A l b$ albumin, $B U N$ blood urea nitrogen, $C c r$ creatinine clearance, $H b A 1 c$ hemoglobin A1c, $C a$ calcium, $P$ phosphate, $i P T H$ intact parathyroid hormone. ${ }^{a}$ Versus Control group, $P<0.05$. bersus DM group, $P<0.05$. ${ }^{\mathrm{c}}$ Versus CKD group, $P<0.05 .{ }^{\mathrm{d}}$ Versus $\mathrm{CKD}+\mathrm{DM}, P<0.05$. ${ }^{\text {e}}$ Versus CKD + DM + INS, $P<0.05$.

In this study, we developed a model rat with both CKD and DM and investigated the mechanisms of VC in the setting of coexisting of CKD and DM, particularly in the context of oxidative stress.

\section{Results}

Animal characteristics and biochemical measurements. The animal characteristics and biochemical data of the rats at 18 weeks old are shown in Table 1 . Creatinine clearance $(\mathrm{Ccr})$ was significantly lower in the CKD and CKD complicated by DM groups than in the control and DM groups and the treatment with INS or APO did not significantly change the kidney function. Blood glucose and HbAlc levels were higher in the $\mathrm{DM}$, the $\mathrm{CKD}+\mathrm{DM}$ and the $\mathrm{CKD}+\mathrm{DM}+\mathrm{APO}$ groups than in the control and CKD groups. These parameters also did not differ significantly between the $\mathrm{CKD}+\mathrm{DM}$ and $\mathrm{CKD}+\mathrm{DM}+\mathrm{APO}$ groups. Serum phosphorus levels were significantly higher and serum calcium levels were significantly lower in the CKD and CKD complicated by DM groups than in the control and DM groups.

Evaluation of aortic calcification. We measured the percentage of the positive areas with von Kossa staining and the Ca contents of the aorta to assess the degree of aortic calcification in each group. Representative photomicrographs of the aorta stained with von Kossa staining are shown in Fig. 1a,b. Compared with the $\mathrm{CKD}$ and $\mathrm{DM}$ groups, the percentage of the positive areas with von Kossa staining was significantly higher in the CKD + DM group (Fig. 1c). However, compared with the CKD + DM group, the CKD + DM + INS and the $\mathrm{CKD}+\mathrm{DM}+\mathrm{APO}$ groups showed less aortic calcification. The presence of CKD and DM also increased the Ca contents of aortas, and the coexistence of these two conditions intensified the degree of calcification (Fig. 1d). Besides the results of the evaluation with von Kossa staining, the CKD + DM + APO group showed less aortic calcification than in the CKD + DM group, despite comparable blood glucose control.

Evaluation of systemic and intraaortic oxidative stress. To evaluate systemic oxidative stress, we measured the urinary excretion of 8-hydroxydeoxyguanosine (8-OHdG). Urinary levels of 8-OHdG were corrected by urinary levels of creatinine for evaluation (Fig. 2). Urinary 8-OHdG excretion was higher in the $\mathrm{DM}, \mathrm{CKD}$, and CKD complicated by DM groups than in the control group (Control, $15.7 \pm 0.8 \mathrm{ng} / \mathrm{mg}$. Cr; $\mathrm{DM}, 39.4 \pm 6.0 \mathrm{ng} / \mathrm{mg} \cdot \mathrm{Cr}$; CKD, $29.6 \pm 6.4 \mathrm{ng} / \mathrm{mg} \cdot \mathrm{Cr})$, and the CKD + DM + INS and CKD + DM + APO groups revealed a marked decrease in oxidative stress compared with the CKD + DM group (CKD + DM, $94.4 \pm 16.7 \mathrm{ng} /$ $\mathrm{mg} \cdot \mathrm{Cr} ; \mathrm{CKD}+\mathrm{DM}+\mathrm{INS}, 38.5 \pm 8.5 \mathrm{ng} / \mathrm{mg} \cdot \mathrm{Cr} ; \mathrm{CKD}+\mathrm{DM}+\mathrm{APO}, 55.7 \pm 6.4 \mathrm{ng} / \mathrm{mg} \cdot \mathrm{Cr})$. We also evaluated oxidative stress in the aortas with immunohistochemical methods. The 8-OHdG formation in aorta was significantly increased in the DM, CKD, and CKD complicated by DM groups compared with the control group (Fig. 3; Control, 1.6 $\pm 0.1 / 0.01 \mathrm{~mm}^{2}$; DM, 5.4 $\pm 0.2 / 0.01 \mathrm{~mm}^{2}$; CKD, 4.2 $\pm 0.1 / 0.01 \mathrm{~mm}^{2}$; CKD + DM, $8.5 \pm 0.3$ $/ 0.01 \mathrm{~mm}^{2} ; \mathrm{CKD}+\mathrm{DM}+\mathrm{INS}, 5.5 \pm 0.2 / 0.01 \mathrm{~mm}^{2}$; CKD + DM + APO, $\left.6.1 \pm 0.2 / 0.01 \mathrm{~mm}^{2}\right)$. The treatment with $\mathrm{APO}$ as well as INS reduced intraaortic oxidative stress compared with the CKD + DM group.

Moreover, the mRNA expression of nicotinamide adenine dinucleotide phosphate (NADPH) oxidase was remarkably increased in the CKD + DM groups compared with the control group (Fig. 4). The expressions of oxidative stress-related markers were also significantly decreased in the CKD +DM + INS and CKD $+D M+A P O$ groups compared with the CKD + DM group.

Evaluation of osteoblast differentiation-related markers. To evaluate the known osteogenic markers related to VC, we evaluated mRNA expression of alkaline phosphatase (ALP) and Runt-related transcription factor 2 (RUNX2) in the aortas. The mRNA expressions of ALP and RUNX2 were higher in the DM, CKD, and three CKD + DM groups than in the control group (Fig. 5). The immunohistochemical staining of RUNX2 also showed the number of RUNX2 positive cells was significantly higher in the CKD+DM group than in the con- 
a

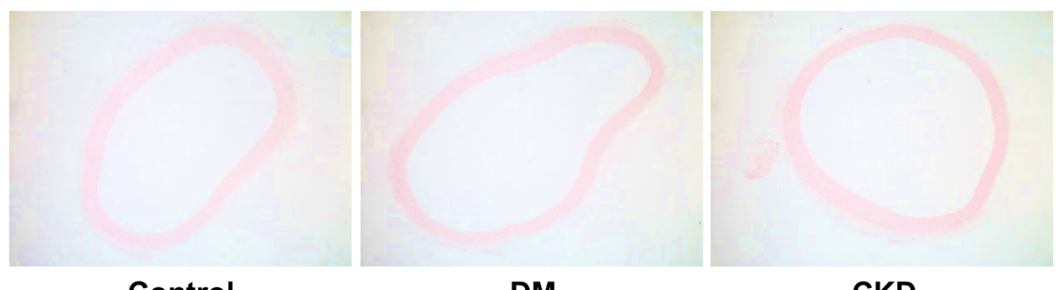

Control

DM

CKD

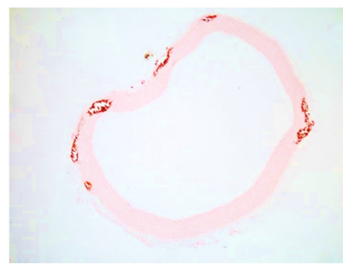

CKD+DM

CKD+DM+INS

CKD+DM+APO

b

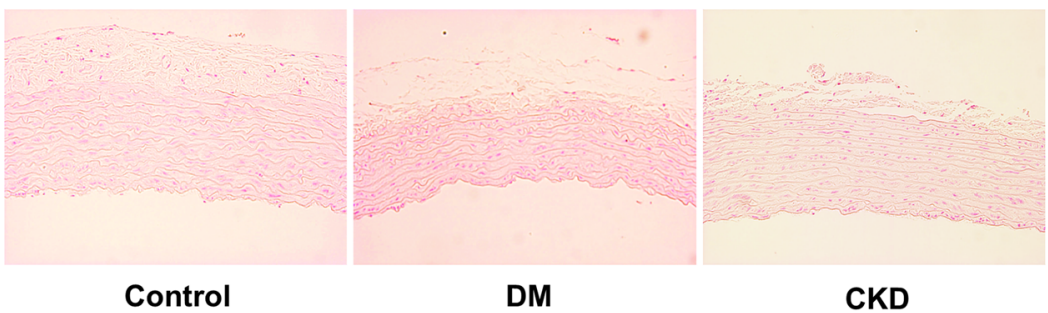

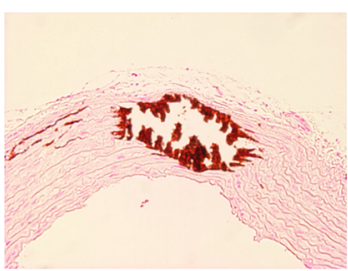

CKD+DM
DM

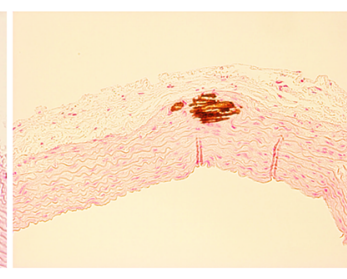

CKD+DM+INS

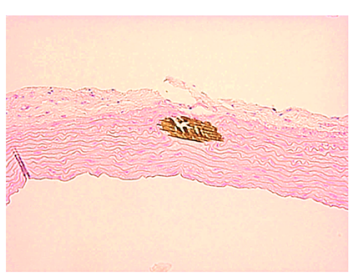

CKD+DM+APO
C

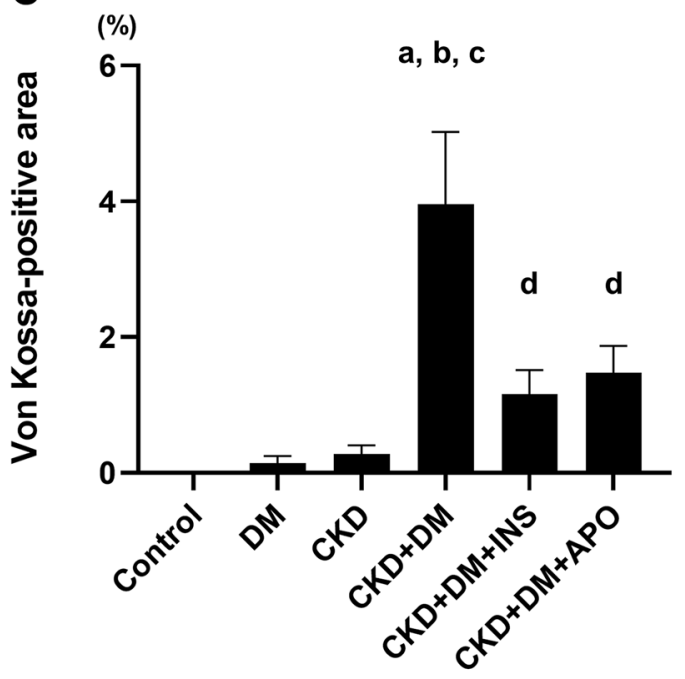

d

( $\mu \mathrm{g} / \mathrm{mg} \cdot \mathrm{dry}$ tissue weight)

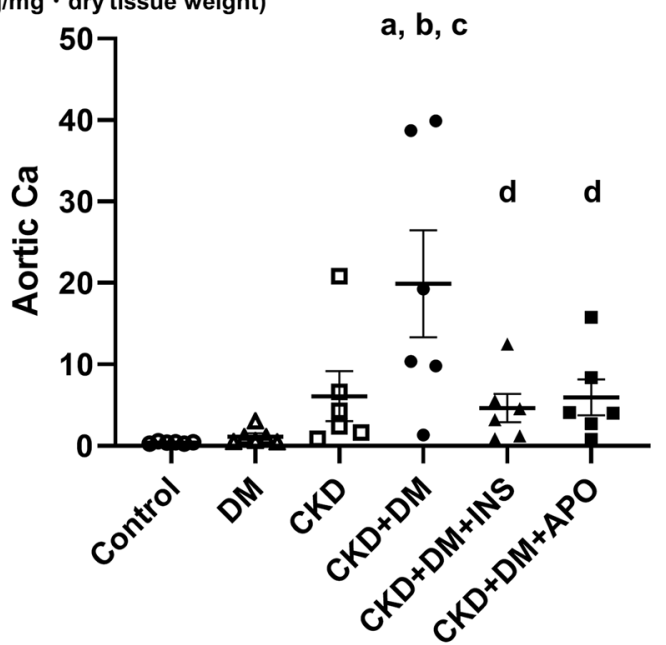

Figure 1. Evaluation of calcification of aortas. (a) Representative photomicrographs of the aorta with von Kossa stain (magnification, $\times 40$ ). (b) Representavive photomicrographs of the aorta with von Kossa stain (magnification, $\times 200$ ). (c) The percentage of von Kossa-positive mineralized area of aortas. (d) Calcium contents of aortas. DM, diabetes mellitus; CKD, chronic kidney disease; INS, insulin; APO, apocynin; Ca, calcium. ${ }^{a}$ Versus Control group, $P<0.05$, ${ }^{\mathrm{b}}$ versus DM group, $P<0.05$, cversus CKD group, $P<0.05$, ${ }^{\mathrm{d}}$ versus $\mathrm{CKD}+\mathrm{DM}$, $P<0.05$. 


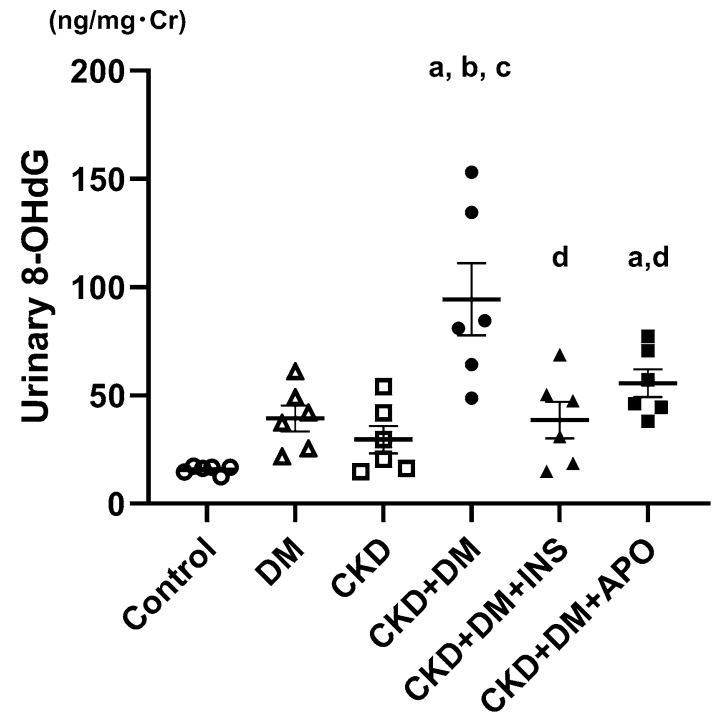

Figure 2. Evaluation of systemic oxidative stress. 8-OHdG, 8-hydroxy-2'-deoxyguanosine; DM, diabetes mellitus; CKD, chronic kidney disease; INS, insulin; APO, apocynin. ${ }^{a}$ versus Control group, $P<0.05$, bersus DM group, $P<0.05$, ${ }^{c}$ versus CKD group, $P<0.05$, ${ }^{\mathrm{d}}$ versus $\mathrm{CKD}+\mathrm{DM}, P<0.05$.

trol, CKD, and DM groups (Fig. 6). Similarly, these expression of osteoblast differentiation-related markers were significantly decreased in the CKD + DM + APO group, as well as in the CKD + DM + INS group, compared with the CKD + DM group.

Relationship between oxidative stress, osteoblast differentiation-related markers, and calcification. We analyzed the correlation of systemic oxidative stress with the RUNX2 expression in the aortas and calcium content of the aorta. Urinary 8 -OHdG excretion was significantly correlated with the mRNA expression of RUNX2 and the number of RUNX2-positive cells in the aortas (Fig. 7a: mRNA expression of RUNX2, $r=0.397, p<0.05$; Fig. 7b: the number of RUNX2-positive cells, $r=0.567, p<0.05$ ). Moreover, urinary 8 -OHdG excretion was also significantly correlated with the calcium content of the aortas (Fig. $7 c$ : $r=0.612, p<0.05$ ).

\section{Discussion}

In this study, we demonstrated that (1) von Kossa-positive mineralized area and calcium content of aortas were higher in the CKD + DM group than in the control, CKD, and DM groups; (2) the expressions of osteoblast differentiation-related markers and oxidative stress-related markers were increased in the CKD + DM group compared with the control, CKD, and DM groups; (3) the degree of VC and oxidative stress was significantly lower in the $\mathrm{CKD}+\mathrm{DM}+\mathrm{APO}$ group than in the CKD + DM group; and (4) oxidative stress-related markers were significantly and positively correlated with osteoblast differentiation-related markers.

$\mathrm{VC}$ is a major risk factor for $\mathrm{CVD}^{22}$, and exploring strategies for the prevention of VC progression is very important. Although DM and CKD are known to play key roles in VC progression, few studies elucidated how their coexistence impacts its progression. Our previous observational study showed that the presence of DM was significantly associated with the degree of aortic calcification in patients with stages 4 and $5 \mathrm{CKD}^{23}$. After adjustment for several confounding factors, DM was one of the most important risk factors for VC in these advanced-stage CKD patients. In patients undergoing hemodialysis, a significantly positive association was observed between hemoglobin Alc levels and the degree of $\mathrm{VC}^{24}$. Although serum phosphate level is recognized as a risk factor for $\mathrm{VC}$, a previous study reported that mineral bone disorder was not significantly associated with VC in diabetic patients undergoing hemodialysis ${ }^{25}$. Considering these results, DM is seen to be a crucial condition for VC progression even in CKD.

The mechanisms underlying VC progression in CKD and DM are complicated, and several factors contribute to its pathogenesis. There are many reports about the mechanisms of VC progression in the field of CKD and DM; however, few studies focused on the VC progression in CKD complicated by DM. Many factors such as hyperlipidemia, hypertension, DM, aging, and mineral bone disorder, are considered to be related to the pathophysiological mechanisms of $\mathrm{VC}^{26,27}$. Among them, we focused on oxidative stress in this study. Many studies have shown that oxidative stress may contribute to the pathogenesis of VC progression in a rat CKD and DM model $^{28,29}$. Our several previous studies also proved that an increase in oxidative stress was associated with the progression of kidney and cardiovascular injury in not only CKD but also $\mathrm{DM}^{16-18,30}$. In this study, both the CKD and DM groups manifested increased oxidative stress and rats with CKD complicated by DM exhibited increased oxidative stress compared with them.

NADPH oxidases are OHdG-generating enzymes and have been implicated in the pathophysiology of various type of organ damage ${ }^{31}$. Studies on the CV system have reported that NADPH oxidases promote endothelial dysfunction, inflammation, cardiac hypertrophy, fibrosis, apoptosis, and remodeling in vessel walls and the 
a

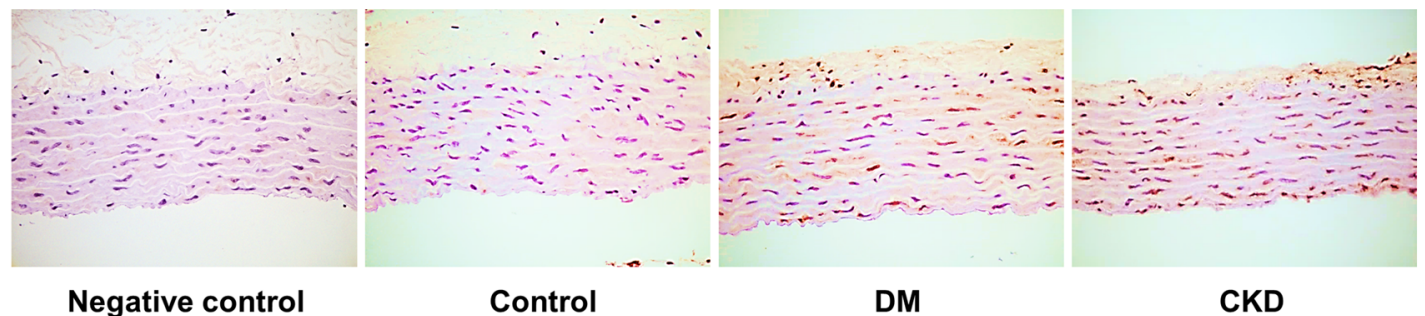

$\begin{array}{llll}\text { Negative control } & \text { Control } & \text { DM }\end{array}$

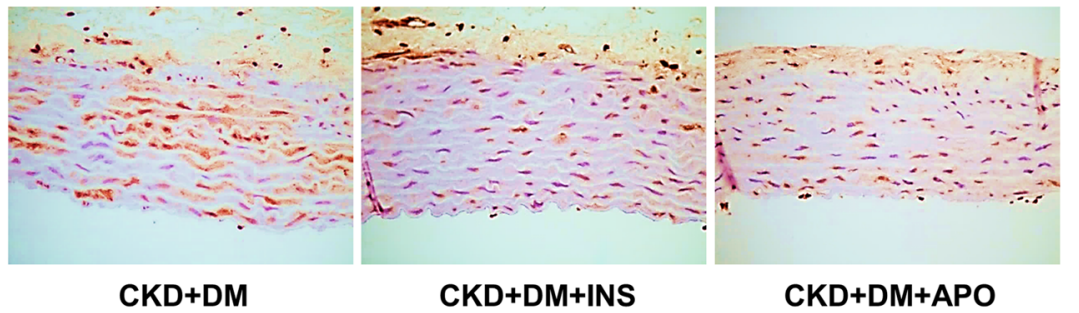

b

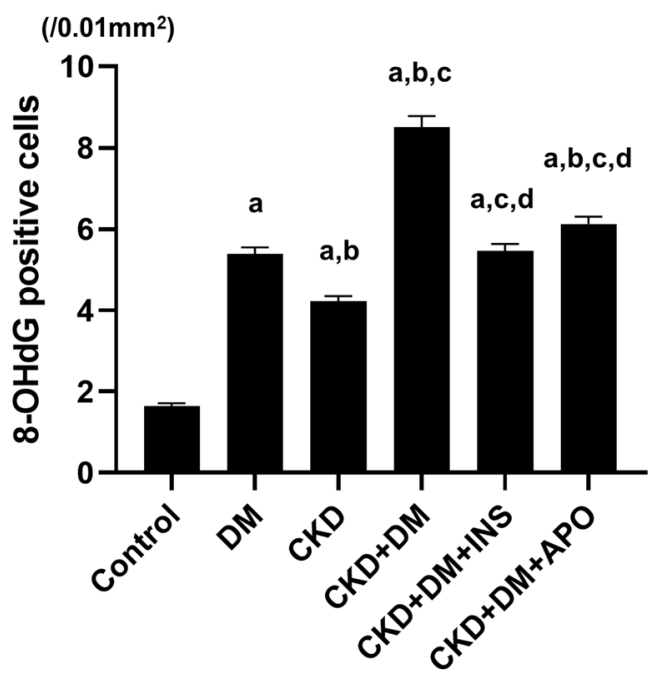

Figure 3. Evaluation of oxidative stress in aortas. (a) Representative photomicrographs of aortas with immunohistochemical staining for 8-OHdG (magnification, $\times 400$ ). (b) The number of 8-OHdG positive cells in aortas. 8-OHdG, 8-hydroxy-2'-deoxyguanosine; DM, diabetes mellitus; $\mathrm{CKD}$, chronic kidney disease; INS,

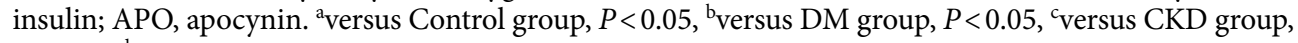
$P<0.05$, ${ }^{\mathrm{d}}$ versus CKD $+\mathrm{DM}, P<0.05$.

heart ${ }^{32,33}$. Our previous experimental study using diabetic model rats demonstrated that the mRNA expression of NADPH oxidase considerably increased in aortic tissue compared with the control rats and glycemic control with insulin suppressed the increase ${ }^{16}$. Moreover, another study using CKD model rats showed that the mRNA expression of NADPH oxidase in aortic tissue and urinary excretion of 8-OHdG increased, and urinary excretion of 8-OHdG was significantly correlated with the degree of $\mathrm{VC}^{28}$. We also found that the mRNA expression of NADPH oxidase significantly increased in aortic tissue in the CKD + DM group compared with the CKD and DM groups. Urinary excretion of 8-OHdG and the expression of $8-\mathrm{OHdG}$ in the aortic tissue also revealed a remarkable increase in the $\mathrm{CKD}+\mathrm{DM}$ group. To our knowledge, the present study is the first to examine oxidative stress in the context of coexisting CKD and DM. In clinical study, it has been reported that hemodialysis patients with DM had significantly higher oxidative albumin levels compared with those without $\mathrm{DM}^{34}$. Another study demonstrated that increased NADPH oxidase-mediated superoxide production was associated with enhanced coronary artery calcification in the general population ${ }^{35}$. Taken together, increased oxidative stress enhanced by both CKD and DM can play a key role in the VC progression.

Regarding the pathogenesis of VC progression, the effect of oxidative stress on vascular smooth muscle cells (VSMCs) is considered to be crucial ${ }^{36-38}$. The formation and accumulation of advanced glycation end products (AGEs) are accelerated in the context of CKD and $\mathrm{DM}^{29,39}$. AGE interacts with the receptor for AGE, leading to vascular injury by evoking oxidative stress ${ }^{40}$. An in vitro study showed that AGE induced calcium deposition in VSMCs through increases in the mRNA expression of NADPH oxidase and 8-OHdG concentration in the medium ${ }^{41}$. Moreover, AGE increased the mRNA expression of osteoblast differentiation-related markers as well as oxidative stress-related markers in VSMCs. Although we could not evaluate AGE, the results of our study also showed that urinary 8-OHdG excretion was significantly correlated with the RUNX2 expression and the calcium content of the aorta. Oxidative stress has been reported to induce the expression of bone markers 
a

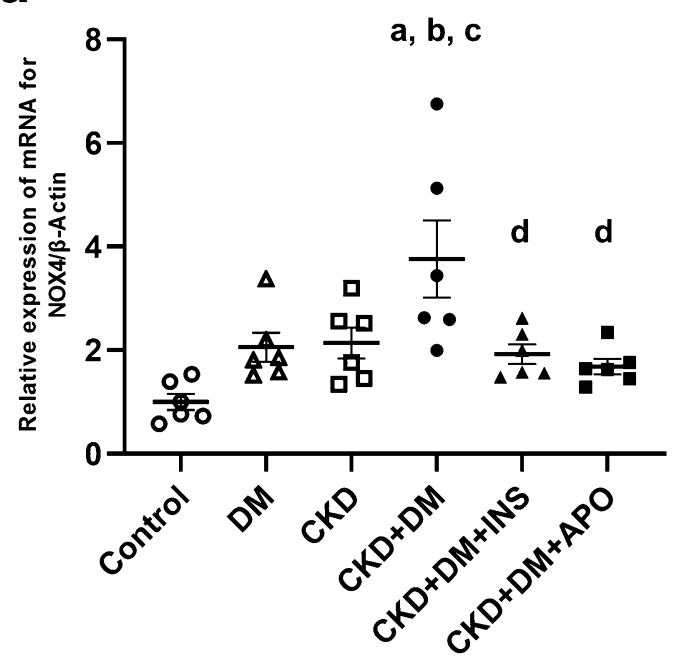

b

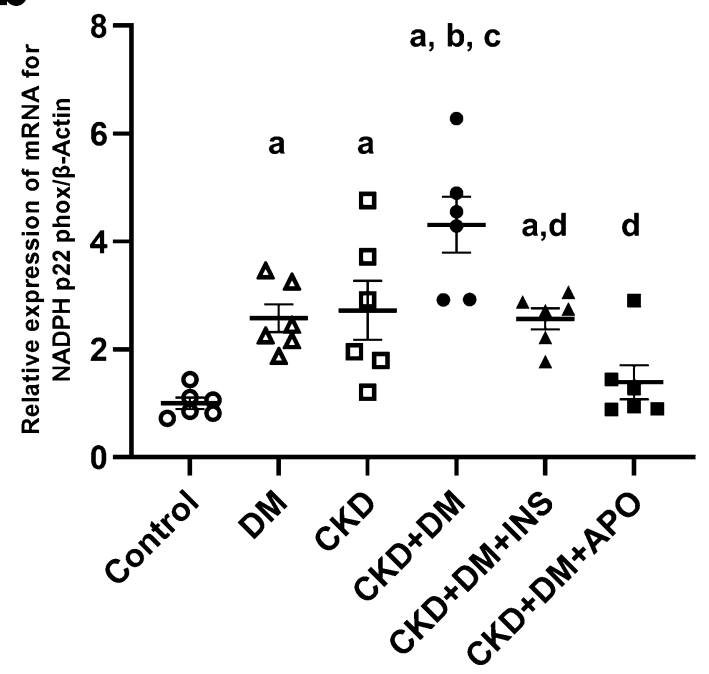

Figure 4. mRNA expression of NADPH oxidase. (a) NOX4. (b) NADPH p22 phox. NOX4, nicotinamide adenine dinucleotide phosphate oxidase 4; NADPH, nicotinamide adenine dinucleotide phosphate oxidase; $\mathrm{DM}$, diabetes mellitus; CKD, chronic kidney disease; INS, insulin; APO, apocynin. "aversus Control group, $P<0.05$, ${ }^{b}$ versus DM group, $P<0.05$, ${ }^{c}$ versus CKD group, $P<0.05$, ${ }^{\mathrm{d}}$ versus $\mathrm{CKD}+\mathrm{DM}, P<0.05$.

\section{a}

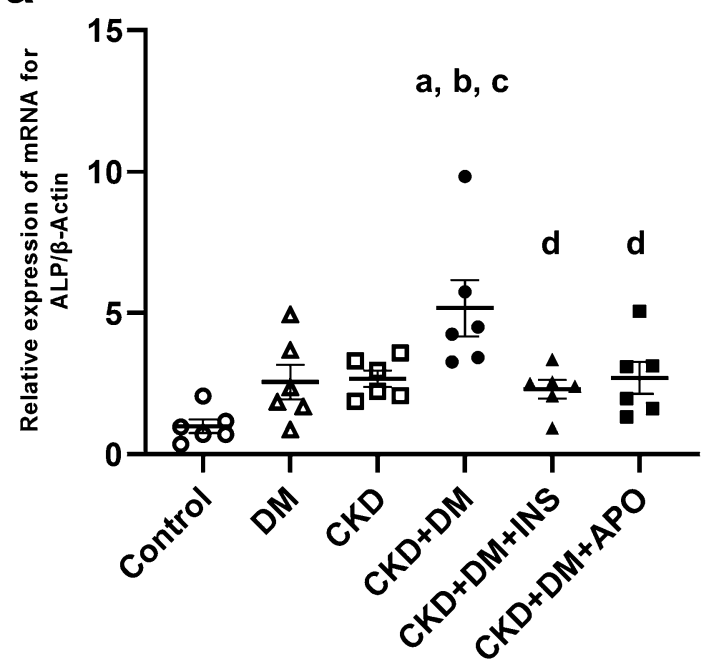

b

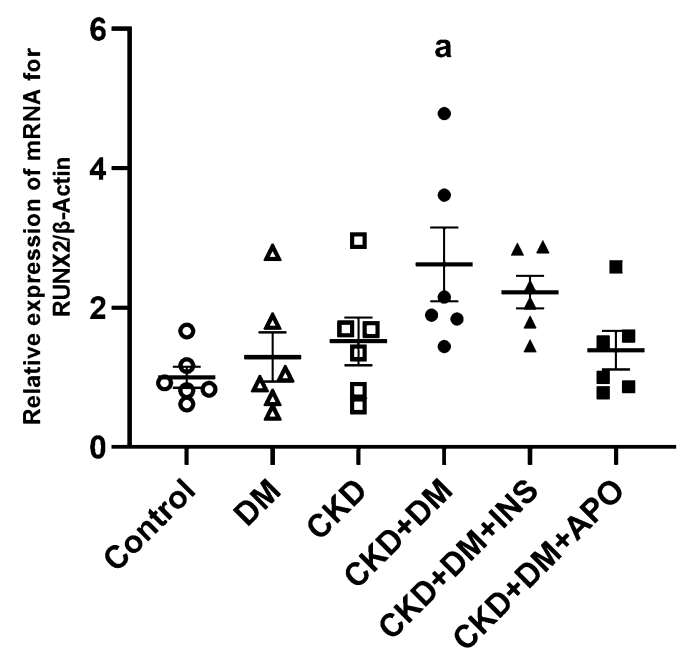

Figure 5. mRNA expression of osteoblast-differentiation markers. (a) ALP. (b) RUNX2. ALP, alkaline phosphatase; RUNX2, Runt-related transcription factor 2; DM, diabetes mellitus; CKD, chronic kidney disease; INS, insulin; APO, apocynin. a versus Control group, $P<0.05$, bersus DM group, $P<0.05$, ${ }^{c}$ versus CKD group, $P<0.05,{ }^{\mathrm{d}}$ versus CKD $+\mathrm{DM}, P<0.05$.

and VSMC calcification through the activation of the AKT signaling pathway and downregulation of VSMC markers $^{36}$. We confirmed that the expressions of ALP and RUNX2 in the aorta were highest in the CKD + DM group. From these results, it was gathered that oxidative stress induced differentiation of VSMC to osteoblast, and leading to VC progression.

From the clinical setting perspective, it has been speculated that the prevention of VC progression can reduce CVD events and mortality. Therefore, a treatment that halts VC progression would be of great interest to many clinicians. The suppression of oxidative stress has been verified as one such treatment. A randomized controlled trial for patients undergoing maintenance hemodialysis demonstrated that the antioxidant acetylcysteine significantly reduced CVD events compared with the placebo ${ }^{42}$. In this study, we used apocynin to examine the effect of antioxidative therapy on VC. Treatment with apocynin decreased aortic calcification and osteoblast differentiation-related markers through the suppression of oxidative stress in the context of coexisting CKD and DM. Since the streptozotocin-induced diabetes is characterized by insulin deficiency, these results could not apply to all diabetic patients. However, impairment of insulin secretion is a more important pathophysiological 

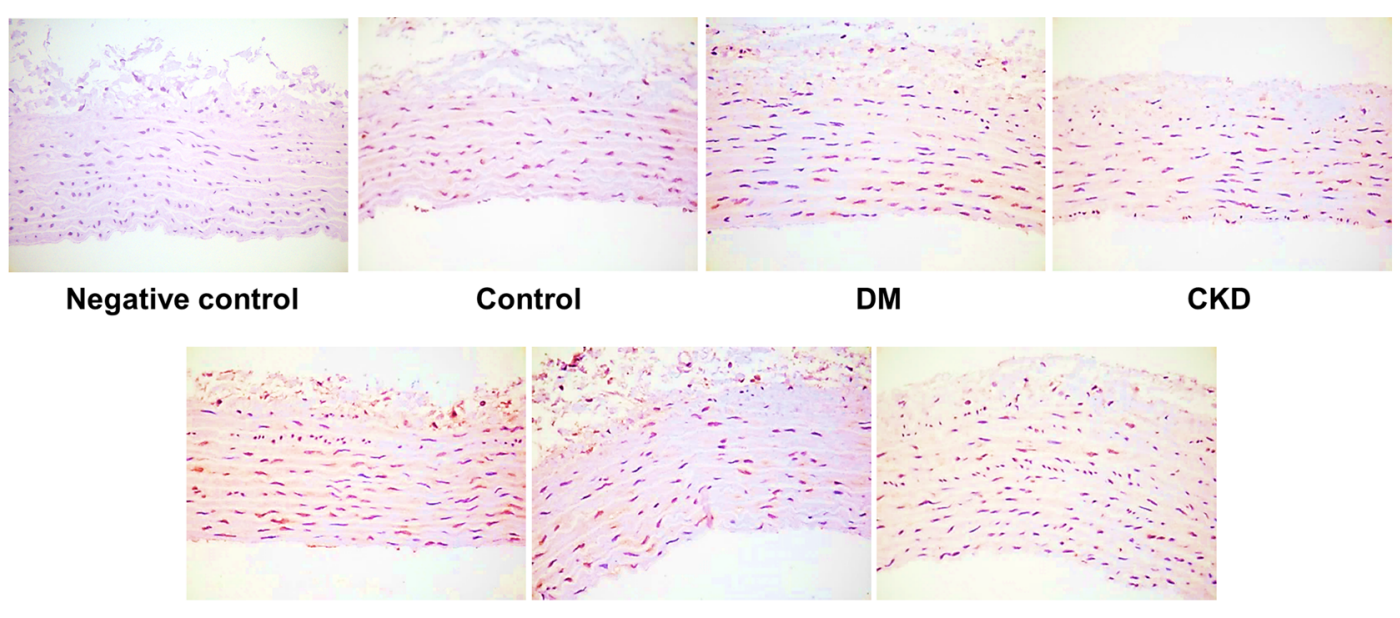

CKD+DM CKD+DM+INS CKD+DM+APO

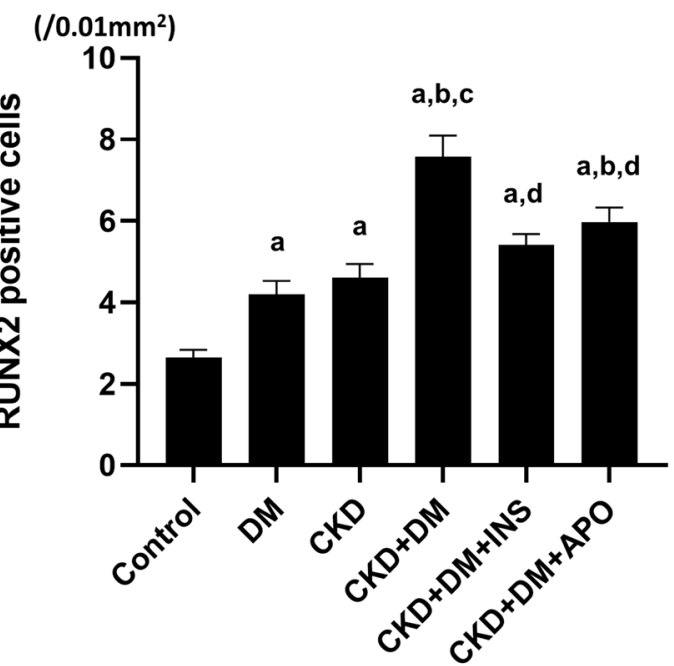

Figure 6. Evaluation of RUNX2 expression with immunohistochemical staining. (a) Representative photomicrographs of aortas with immunohistochemical staining for RUNX2 (magnification, $\times 400$ ). (b) The number of RUNX2 positive cells in aortas. RUNX2, Runt-related transcription factor 2; DM, diabetes mellitus; $\mathrm{CKD}$, chronic kidney disease; INS, insulin; APO, apocynin. ${ }^{\mathrm{a}}$ versus Control group, $P<0.05$, bersus DM group, $P<0.05$, ${ }^{c}$ versus CKD group, $P<0.05$, ${ }^{\mathrm{d}}$ versus $\mathrm{CKD}+\mathrm{DM}, P<0.05$.

condition in type 1 diabetic and Asian type 2 diabetic patients. Many patients with type 2 diabetes in Asia often show non-obesity and impairment of insulin secretion. Therefore, we think the results of the present study could be meaningful particularly for such diabetic conditions.

Our data suggest that antioxidative therapy could be useful for the prevention of VC progression in CKD and DM. To ascertain clinical efficacy, further clinical study is needed.

\section{Methods}

Animals and experimental protocol. Six-week-old male Sprague Dawley (SD) rats were obtained from CLEA Japan Inc. (Tokyo, Japan). The rats were housed with ad libitum access to food and water and in lightand temperature-controlled environments. At 7 weeks old, the rats were randomly divided into four groups as follows; sham-operated SD (control, $n=6$ ), sham-operated and induced-DM SD (DM, $n=6)$, induced-CKD SD $(\mathrm{CKD}, \mathrm{n}=6)$, and induced-CKD and induced-DM SD (CKD complicated by DM, $\mathrm{n}=18$ ).

At 7 weeks age, the rats in the CKD and CKD complicated by DM groups were briefly anesthetized with sodium pentobarbital and underwent two-thirds nephrectomies of the right kidneys. One week later, the remaining left kidneys were resected. The rats in the control and DM group underwent sham operations at the same time. At 9 weeks old, rats in the DM and CKD complicated by DM groups were injected, under anesthesia, with streptozotosin (STZ; $55 \mathrm{mg} / \mathrm{kg}$ body weight, Sigma Chemical Co., St louis, MO, USA) dissolved in $0.1 \mathrm{~mol} / \mathrm{l}$ sodium citrate buffer, $\mathrm{pH} 4.5$, via tail veins. The rats in the control and CKD groups were injected with an equal amount of citrate buffer alone. One week later, tail vein blood glucose was determined using a Glutest Ace (Sanwa Kagaku Kenkyusho, Nagoya, Japan). Rats treated with STZ were considered to have DM when blood glucose levels were higher than $300 \mathrm{mg} / \mathrm{dl}$. At 11 weeks old, the rats in the CKD complicated by DM group were further divided into three groups as follows: the rats with no treatment $(\mathrm{CKD}+\mathrm{DM}, \mathrm{n}=6)$, the rats treated with insulin 

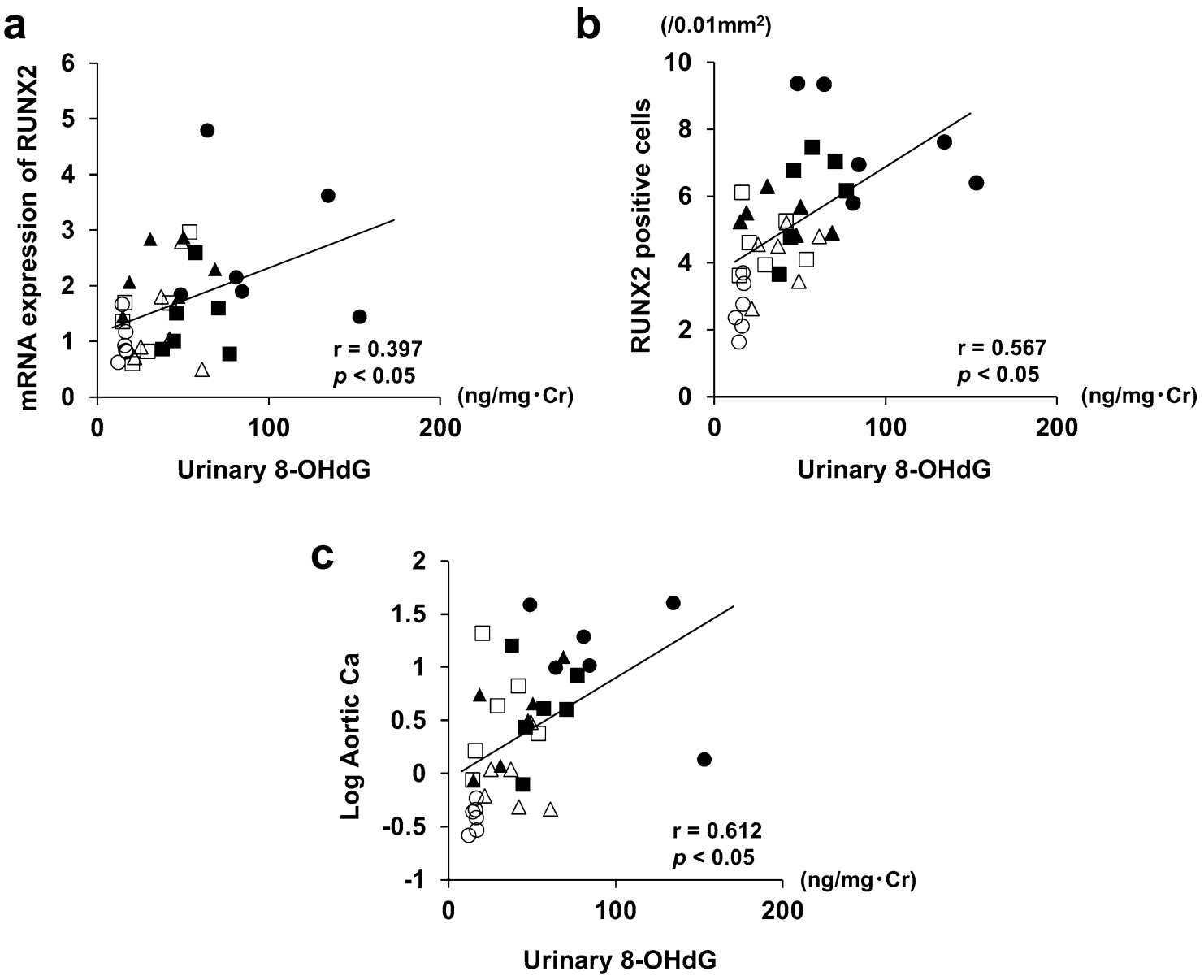

Figure 7. Correlation of oxidative stress with RUNX2 and calcification. (a) Relationship between urinary 8-OHdG excretion and mRNA expression of RUNX2 in aortas. Urinary 8-OHdG excretion was significantly associated with mRNA expression of RUNX2 $(r=0.397, P<0.05)$. (b) Relationship between urinary 8-OHdG excretion and the number of RUNX2 positive cells in aortas. Urinary 8-OHdG excretion was significantly associated with the number of RUNX2 positive cells in aortas $(r=0.567, P<0.05)$. (c) Relationship between urinary 8-OHdG excretion and calcium contents of aortas. Urinary 8-OHdG excretion was significantly associated with calcium contents of aortas $(r=0.612, P<0.05)$. Symbols denote the same group in each figure (Control group, open circle; DM group, open triangle; CKD group, open square; CKD + DM group, closed circle; $\mathrm{CKD}+\mathrm{DM}+\mathrm{INS}$ group, closed triangle; CKD + DM + APO group, closed square). 8-OHdG, 8-hydroxy-2'deoxyguanosine; RUNX2, Runt-related transcription factor 2; Ca, calcium.

$(\mathrm{CKD}+\mathrm{DM}+\mathrm{INS}, \mathrm{n}=6)$, and the rats treated with an antioxidant agent, apocynin $(\mathrm{CKD}+\mathrm{DM}+\mathrm{APO}, \mathrm{n}=6)$. Lastly, the rats were divided into six groups as described above.

At 11 weeks old, the rats in the CKD + DM + INS group were treated with insulin implants (LinShin Canada Inc., Scarborough, Ont., Canada), which release a controlled amount of insulin. The rats in the CKD $+\mathrm{DM}+\mathrm{APO}$ group were given drinking water containing three mmol/l apocynin (Tokyo Chemical Industry, Co., Ltd., Tokyo, Japan) until the end of the study period. Apocynin has been used in experimental studies as an antioxidant agent and inhibits NADPH oxidases and scavenges reactive oxygen species (ROS) ${ }^{43}$.

The rats in all groups were fed a high phosphate diet containing $1.0 \%$ calcium and $2.0 \%$ phosphate. Before the rats were sacrificed, $24 \mathrm{~h}$ urine samples were collected from each rat using a metabolic cage. At 18 weeks old, these rats were sacrificed under sodium pentobarbital anesthesia. Blood samples were collected from the left ventricles for serum biochemical analysis, and the aortas were removed for RNA extraction and histomorphological analysis.

This study was conducted in strict accordance with the recommendations in the Guide for the Care and Use of Laboratory Animals of the National Institutes of Health. The Institutional Animal Care and Use Committee at Kobe University Graduate School of Medicine approved the protocol (Permit Number: P150507). All surgeries were conducted under sodium pentobarbital anesthesia, and every effort was made to minimize suffering, following the ARRIVE guidelines for reporting experiments involving animals ${ }^{44}$.

Blood and urine analysis. After $3000 \mathrm{rpm}$ centrifugation for $10 \mathrm{~min}$, the serum and urine samples were stored at $-80^{\circ} \mathrm{C}$ until analysis. Serum creatinine $(\mathrm{Cr})$ and urea nitrogen levels were measured using Fuji DriChem 3500 (Fujifilm Japan, Tokyo, Japan). Urine $\mathrm{Cr}$ and serum calcium and phosphate levels were measured at 
SRL Inc (Tokyo, Japan). HbA1c levels were determined with a DCA 2000 analyzer (Bayel Medical, Tokyo, Japan), and glucose levels were determined using a Glutest Ace with venous blood samples obtained from tails. As a sensitive marker of oxidative stress, the urinary excretion of 8-OHdG was detected using an ELISA kit (Japan Institute for Control of Aging, Shizuoka, Japan). The plasma parathyroid hormone levels were measured using an ELISA kit (Immunotopics, San Clemente, CA, USA).

Blood pressure measurements. Systolic blood pressure was measured by tail-cuff plethysmography (Model MK-2000; Muromachi Kikai CO., Ltd., Tokyo, Japan). To reduce the possibility of stress artifacts, the measurements were taken after a sufficient acclimatization period. Systolic blood pressure was determined by multiple readings for each rat at baseline and the end of the study period.

Evaluation of vascular calcification. To quantify calcium content, the lower parts of the abdominal aortas were removed. The aortas were freeze-dried, weighed, and then incubated in $0.6 \mathrm{~N} \mathrm{HCl}$ for $48 \mathrm{~h}$. The samples were centrifuged and the supernatant were analyzed for calcium contents using the Calcium-E test (Wako, Osaka, Japan). Aortic calcium content was corrected for the dry tissue weight. The thoracic aortas were removed and fixed in $10 \%$ formaldehyde for histopathology. These sections were dehydrated with $70 \%$ ethanol at room temperature and embedded in paraffin blocks, which were deparaffinized and processed for von Kossa staining. The percentage of the positive areas of von Kossa staining in the aortic sections were calculated in 30 randomly selected microscopic fields using Lumina Vision image analysis software version 3.7.4.2 (Mitani Co., Tokyo, Japan). All evaluations were conducted in a blinded manner.

Immunohistochemical analyses. The thoracic aortas in paraffin blocks were deparaffinized and processed for immunohistochemical staining. The formation of 8-OHdG was evaluated with anti-8-OHdG monoclonal antibodies raised in rats (Japan Institute for Control of Aging, Shizuoka, Japan). The expression of RUNX2, which is a marker of osteoblast differentiation, was evaluated with anti-RUNX2 antibody (Abcam, Cambridge, United Kingdom). Sectioning and staining were conducted according to standard procedure of Morpho Technology (Sapporo, Japan). To give 8-OHdG-positive and RUNX2-positive cell scores, the 8-OHdG-positive and RUNX2-positive cells in the aortic tissue were counted in 30 random microscopic fields. The results of the positive cells were expressed as the number of positive cells per $0.01 \mathrm{~mm}^{2}$ of the examined area.

RNA extraction and real-time polymerase chain reaction. The upper parts of the abdominal aortas were removed and snap-frozen using liquid nitrogen. Then, these samples were then stored at $-80^{\circ} \mathrm{C}$ until evaluation with real-time polymerase chain reaction (PCR). Using an ISOGEN kit (Wako Pure Chemicals Industries, Ltd., Osaka, Japan), total RNA was extracted from the samples of aortas according to the manufacturer's instructions. Total RNA from the rat aortas was used as the template for cDNA synthesis with the ReveTra ACE qPCR RT kit (TOYOBO Co., Ltd., Osaka, Japan) using an oligo-dT primer as per the manufacturer's recommended protocol. The synthesized cDNA was stored at $-80^{\circ} \mathrm{C}$ until analysis with quantitative PCR. mRNA expression was examined with real-time PCR using the Light Cycler 350 s Real-Time PCR System (Roche Diagnostics, Mannheim, Germany) with the SYBR Green Assay with Thunderbird SYBR qPCR Mix (TOYOBO Co., Ltd., Osaka, Japan) following the manufacturer's protocol. The analysis was performed with the second derivative maximum method of the LightCycler software (version. 4.0; Roche). The relative amount of the sample mRNA was normalized to the $\beta$-actin mRNA. For PCR analysis, we used the following primers; rat NADPH oxidase 4 (NOX4; 5'-GAACCCAAGTTCCAAGCTCA-3', 5'-GCACAAAGGTCCAGAAATCC-3'), rat NADPH p22 phox (5'-GGTGAGCAGTGGACTCCCATT-3', 5'-TGGTAGGTGGCTGCTTGATG-3'), rat Runt-related transcription factor 2 (RUNX2; 5'-AAGTGCGGTGCAAACTTTCT-3', 5'-TCTCGGTGGCTGGTAGTG-3'), rat Alkaline Phosphatase (ALP; 5'-TCCGTGGGTCGGATTCCT-3', 5'-GCCGGCCCAAGAGAGAAA-3'), rat $\beta$-Actin (5'-TGACAGGATGCAGAAGGAGA-3', 5'-TAGAGCCACCAATCCACACA-3').

Statistical analysis. All data are expressed as mean \pm SEM. One-way analysis of variance, followed by the Tukey-Kramer test, was used for comparison of each group. Pearson's correlation coefficient or Spearman rank correlation coefficient was used to analyze relationships between variables as appropriate. A $p$ value of $<0.05$ was considered statistically significant. All statistical analyses were conducted using IBM SPSS Statistics version 23.0 (Chicago, IL, USA).

\section{Data availability}

The datasets generated during and/or analysed during the current study are available from the corresponding author on reasonable request.

Received: 13 June 2020; Accepted: 26 October 2020

Published online: 26 November 2020

\section{References}

1. Hanafusa, N., Nakai, S., Iseki, K. \& Tsubakihara, Y. Japanese society for dialysis therapy renal data registry-a window through which we can view the details of Japanese dialysis population. Kidney Int. Suppl. 5, 15-22. https://doi.org/10.1038/kisup.2015.5 (2015).

2. Go, A. S., Chertow, G. M., Fan, D., McCulloch, C. E. \& Hsu, C. Y. Chronic kidney disease and the risks of death, cardiovascular events, and hospitalization. N. Engl. J. Med. 351, 1296-1305 (2004). 
3. Keith, D. S., Nichols, G. A., Gullion, C. M., Brown, J. B. \& Smith, D. H. Longitudinal follow-up and outcomes among a population with chronic kidney disease in a large managed care organization. Arch. Intern. Med. 164, 659-663 (2004).

4. Foley, R. N., Parfrey, P. S. \& Sarnak, M. J. Epidemiology of cardiovascular disease in chronic renal disease. J. Am. Soc. Nephrol. 9 , $16-23$ (1998).

5. Liu, M. et al. Cardiovascular disease and its relationship with chronic kidney disease. Eur. Rev. Med. Pharmacol. Sci. 18, 2918-2926 (2014).

6. Watanabe, R., Lemos, M. M., Manfredi, S. R., Draibe, S. A. \& Canziani, M. E. Impact of cardiovascular calcification in nondialyzed patients after 24 months of follow-up. Clin. J. Am. Soc. Nephrol. 5, 189-194. https://doi.org/10.2215/CJN.06240909 (2010).

7. Ohta, M., Babazono, T., Uchigata, Y. \& Iwamoto, Y. Comparison of the prevalence of chronic kidney disease in Japanese patients with Type 1 and Type 2 diabetes. Diabet. Med. 27, 1017-1023. https://doi.org/10.1111/j.1464-5491.2010.03049.x (2010).

8. Foley, R. N. et al. Chronic kidney disease and the risk for cardiovascular disease, renal replacement, and death in the United States Medicare population, 1998 to 1999. J. Am. Soc. Nephrol. 16, 489-495. https://doi.org/10.1681/ASN.2004030203 (2005).

9. Tonelli, M. et al. Risk of coronary events in people with chronic kidney disease compared with those with diabetes: a populationlevel cohort study. Lancet 380, 807-814. https://doi.org/10.1016/S0140-6736(12)60572-8 (2012).

10. Manzoor, S. et al. Progression of medial arterial calcification in CKD. Kidney Int. Rep. 3, 1328-1335. https://doi.org/10.1016/j. ekir.2018.07.011 (2018).

11. Cottone, S. et al. Oxidative stress, inflammation and cardiovascular disease in chronic renal failure. J. Nephrol. 21, 175-179 (2008).

12. Shah, S. V., Baliga, R., Rajapurkar, M. \& Fonseca, V. A. Oxidants in chronic kidney disease. J. Am. Soc. Nephrol. 18, 16-28. https ://doi.org/10.1681/ASN.2006050500 (2007).

13. Prabhakar, S., Starnes, J., Shi, S., Lonis, B. \& Tran, R. Diabetic nephropathy is associated with oxidative stress and decreased renal nitric oxide production. J. Am. Soc. Nephrol. 18, 2945-2952. https://doi.org/10.1681/ASN.2006080895 (2007).

14. Usberti, M. et al. Oxidative stress and cardiovascular disease in dialyzed patients. Nephron 91, 25-33. https://doi.org/10.1159/00005 7601 (2002).

15. Bayés, B., Pastor, M. C., Bonal, J., Foraster, A. \& Romero, R. Oxidative stress, inflammation and cardiovascular mortality in haemodialysis-role of seniority and intravenous ferrotherapy: analysis at 4 years of follow-up. Nephrol. Dial. Transplant. 21, 984-990. https://doi.org/10.1093/ndt/gfi294 (2006).

16. Kono, K. et al. Anti-oxidative effect of vitamin $\mathrm{D}$ analog on incipient vascular lesion in non-obese type 2 diabetic rats. Am. J. Nephrol. 37, 167-174. https://doi.org/10.1159/000346808 (2013).

17. Fujii, H. et al. The vitamin D receptor activator maxacalcitol provides cardioprotective effects in diabetes mellitus. Cardiovasc. Drugs Ther. 29, 499-507. https://doi.org/10.1007/s10557-015-6629-y (2015).

18. Nakai, K. et al. Vitamin D activates the Nrf2-Keap1 antioxidant pathway and ameliorates nephropathy in diabetic rats. Am. J. Hypertens. 27, 586-595. https://doi.org/10.1093/ajh/hpt160 (2014).

19. Goto, S. et al. Carvedilol ameliorates low-turnover bone disease in non-obese type 2 diabetes. Am. J. Nephrol. 34, 281-290. https ://doi.org/10.1159/000330853 (2011).

20. Asaba, K. et al. Effects of NADPH oxidase inhibitor in diabetic nephropathy. Kidney Int. 67, 1890-1898. https://doi.org/10.111 $1 / j .1523-1755.2005 .00287 . x(2005)$.

21. Luan, J. et al. Renal protection of in vivo administration of tempol in streptozotocin-induced diabetic rats. J. Pharmacol. Sci. 119, 167-176. https://doi.org/10.1254/jphs.12002FP (2012).

22. Detrano, R. et al. Coronary calcium as a predictor of coronary events in four racial or ethnic groups. N. Engl. J. Med. 358, 1336-1345 (2008).

23. Goto, S. et al. Association between AST-120 and abdominal aortic calcification in predialysis patients with chronic kidney disease. Clin. Exp. Nephrol. 17, 365-371. https://doi.org/10.1007/s10157-012-0717-0 (2013).

24. Ishimura, E. et al. Different risk factors for peripheral vascular calcification between diabetic and non-diabetic haemodialysis patients-importance of glycaemic control. Diabetologia 45, 1446-1448. https://doi.org/10.1007/s00125-002-0920-8 (2002).

25. Taniwaki, H. et al. Aortic calcification in haemodialysis patients with diabetes mellitus. Nephrol. Dial. Transplant. 20, 2472-2478. https://doi.org/10.1093/ndt/gfi039 (2005).

26. Yahagi, K. et al. Pathology of human coronary and carotid artery atherosclerosis and vascular calcification in diabetes mellitus. Arterioscler. Thromb. Vasc. Biol. 37, 191-204. https://doi.org/10.1161/ATVBAHA.116.306256 (2017).

27. Yamada, S. \& Giachelli, C. M. Vascular calcification in CKD-MBD: Roles for phosphate, FGF23, and Klotho. Bone 100, 87-93. https://doi.org/10.1016/j.bone.2016.11.012 (2017).

28. Yamada, S. et al. The antioxidant tempol ameliorates arterial medial calcification in uremic rats: important role of oxidative stress in the pathogenesis of vascular calcification in chronic kidney disease. J. Bone Miner. Res. 27, 474-485. https://doi.org/10.1002/ jbmr.539 (2012).

29. Wei, Q. et al. Advanced glycation end products accelerate rat vascular calcification through RAGE/oxidative stress. BMC Cardiovasc. Disord. 13, 13. https://doi.org/10.1186/1471-2261-13-13 (2013).

30. Fujii, H. et al. Oral charcoal adsorbent (AST-120) prevents progression of cardiac damage in chronic kidney disease through suppression of oxidative stress. Nephrol. Dial. Transplant. 24, 2089-2095. https://doi.org/10.1093/ndt/gfp007 (2009).

31. Pendyala, S. \& Natarajan, V. Redox regulation of Nox proteins. Respir. Physiol. Neurobiol. 174, 265-271. https://doi.org/10.1016/j. resp.2010.09.016 (2010).

32. Murdoch, C. E., Zhang, M., Cave, A. C. \& Shah, A. M. NADPH oxidase-dependent redox signalling in cardiac hypertrophy, remodelling and failure. Cardiovasc. Res. 71, 208-215. https://doi.org/10.1016/j.cardiores.2006.03.016 (2006).

33. García-Redondo, A. B., Aguado, A., Briones, A. M. \& Salaices, M. NADPH oxidases and vascular remodeling in cardiovascular diseases. Pharmacol. Res. 114, 110-120. https://doi.org/10.1016/j.phrs.2016.10.015 (2016).

34. Mera, K. et al. Oxidation and carboxy methyl lysine-modification of albumin: possible involvement in the progression of oxidative stress in hemodialysis patients. Hypertens. Res. 28, 973-980. https://doi.org/10.1291/hypres.28.973 (2005).

35. Beloqui, O. et al. Increased phagocytic NADPH oxidase activity associates with coronary artery calcification in asymptomatic men. Free Radic. Res. 51, 389-396. https://doi.org/10.1080/10715762.2017.1321745 (2017).

36. Byon, C. H. et al. Oxidative stress induces vascular calcification through modulation of the osteogenic transcription factor Runx2 by AKT signaling. J. Biol. Chem. 283, 15319-15327 (2008).

37. Mody, N., Parhami, F., Sarafian, T. A. \& Demer, L. L. Oxidative stress modulates osteoblastic differentiation of vascular and bone cells. Free Radic. Biol. Med. 31, 509-519. https://doi.org/10.1016/S0891-5849(01)00610-4 (2001).

38. Csiszar, A., Lehoux, S. \& Ungvari, Z. Hemodynamic forces, vascular oxidative stress, and regulation of BMP-2/4 expression. Antioxid. Redox Signal. 11, 1683-1697. https://doi.org/10.1089/ars.2008.2401 (2009).

39. Wang, C. C. et al. Spironolactone ameliorates endothelial dysfunction through inhibition of the AGE/RAGE axis in a chronic renal failure rat model. BMC Nephrol. 20, 351. https://doi.org/10.1186/s12882-019-1534-4 (2019).

40. Yamagishi, S. Role of advanced glycation end products (AGEs) and receptor for AGEs (RAGE) in vascular damage in diabetes. Exp. Gerontol. 46, 217-224. https://doi.org/10.1016/j.exger.2010.11.007 (2011).

41. Tada, Y. et al. Advanced glycation end products-induced vascular calcification is mediated by oxidative stress: functional roles of NAD(P)H-oxidase. Horm. Metab. Res. 45, 267-272 (2013). 
42. Tepel, M., van der Giet, M., Statz, M., Jankowski, J. \& Zidek, W. The antioxidant acetylcysteine reduces cardiovascular events in patients with end-stage renal failure: a randomized, controlled trial. Circulation 107, 992-995. https://doi.org/10.1161/01.CIR.00000 50628.11305.30 (2003)

43. Petrônio, M. S., Zeraik, M. L., Fonseca, L. M. \& Ximenes, V. F. Apocynin: chemical and biophysical properties of a NADPH oxidase inhibitor. Molecules 18, 2821-2839. https://doi.org/10.3390/molecules18032821 (2013).

44. Kilkenny, C., Browne, W. J., Cuthill, I. C., Emerson, M. \& Altman, D. G. Improving bioscience research reporting: the ARRIVE guidelines for reporting animal research. PLoS Biol. 8, e1000412. https://doi.org/10.1371/journal.pbio.1000412 (2010).

\section{Author contributions}

S.W. analyzed the data and wrote the manuscript. H.F. contributed to and was responsible for study design, analyzed the data, and the contents of manuscript. K.K., S.G., and K.W. participated in the interpretation of the results and the analysis of data. S.N. contributed to the supervision or mentorship. All authors reviewed and approved the final manuscript.

\section{Competing interests}

The authors declare no competing interests.

\section{Additional information}

Correspondence and requests for materials should be addressed to H.F.

Reprints and permissions information is available at www.nature.com/reprints.

Publisher's note Springer Nature remains neutral with regard to jurisdictional claims in published maps and institutional affiliations.

(c) (i) Open Access This article is licensed under a Creative Commons Attribution 4.0 International cc) License, which permits use, sharing, adaptation, distribution and reproduction in any medium or format, as long as you give appropriate credit to the original author(s) and the source, provide a link to the Creative Commons licence, and indicate if changes were made. The images or other third party material in this article are included in the article's Creative Commons licence, unless indicated otherwise in a credit line to the material. If material is not included in the article's Creative Commons licence and your intended use is not permitted by statutory regulation or exceeds the permitted use, you will need to obtain permission directly from the copyright holder. To view a copy of this licence, visit http://creativecommons.org/licenses/by/4.0/.

(C) The Author(s) 2020 